Review

\title{
Lethal (2) Giant Larvae: An Indispensable Regulator of Cell Polarity and Cancer Development
}

\author{
Fang $\mathrm{Cao}^{1}$, Yi Miao', Kedong $\mathrm{Xu}^{2}$, Peijun Liu ${ }^{1}{ }^{\square}$ \\ 1. Center for Translational Medicine, The First Affiliated Hospital of Xian Jiaotong University, College of Medicine, Xi'an, China; \\ 2. Department of Hepatobiliary Surgery, The First Affiliated Hospital of Xian Jiaotong University, College of Medicine, Xi'an, China
}

$\triangle$ Corresponding author: Center for Translational Medicine, The First Affiliated Hospital of Xian Jiaotong University, College of Medicine, Xi' an, Shaan Xi Province, China, 710061.Tel.: +86-18991232306; E-mail address: liupeijun@mail.xjtu.edu.cn (P. Liu)

(C) 2015 Ivyspring International Publisher. Reproduction is permitted for personal, noncommercial use, provided that the article is in whole, unmodified, and properly cited. See http://ivyspring.com/terms for terms and conditions.

Received: 2014.12.05; Accepted: 2015.01.21; Published: 2015.02.15

\begin{abstract}
Cell polarity is one of the most basic properties of all normal cells and is essential for regulating numerous biological processes. Loss of polarity is considered a hallmark for cancer. Multiple polarity proteins are implicated in maintenance of cell polarity. Lethal (2) giant larvae (Lgl) is one of polarity proteins that plays an important role in regulating cell polarity, asymmetric division as well as tumorigenesis. Lgl proteins in different species have similar structures and conserved functions. $\mathrm{Lgl}$ acts as an indispensable regulator of cell biological function, including cell polarity and asymmetric division, through interplaying with other polarity proteins, regulating exocytosis, mediating cytoskeleton and being involved in signaling pathways. Furthermore, Lgl plays a role of a tumor suppressor, and the aberrant expression of Hugl, a human homologue of Lgl, contributes to multiple cancers. However, the exact functions of $\mathrm{Lgl}$ and the underlying mechanisms remain enigmatic. In this review, we will give an overview of the Lgl functions in cell polarity and cancer development, discuss the potential mechanisms underlying these functions, and raise our conclusion of previous studies and points of view about the future studies.
\end{abstract}

Key words: lethal (2) giant larvae, cell polarity, asymmetric division, tumor suppression

\section{Introduction}

Lethal (2) giant larvae ( $\mathrm{Lgl})$ proteins, a group of conserved proteins, plays a crucial role in regulating cell polarity. Cell polarity is a cell property of asymmetric distribution of proteins, lipids, carbohydrates and RNA macromolecules. This property is crucial for a multitude of cellular fates, including differentiation, proliferation, migration, adhesion and transformation $[1,2]$.

Lgl mainly locates in apical and basolateral domains, thus plays an important role in regulating apico-basal polarity. Apico-basal polarity, dividing the cell into the apical and basal domains, defines the structural and functional polarity of an epithelial cell, which is essential for diverse cellular processes, including cell proliferation, differentiation, asymmetric cell division, cell migration, tissue morphogenesis, and tumor formation. Many excellent reviews have covered this subject in recent years [3-5]. As we know, apico-basal polarity is regulated by several conserved polarity proteins. These proteins control asymmetric cell division by regulating the polarized localization of cell fate determinants and maintaining the correct orientation of mitotic spindles in different tissues and organisms [6-8]. These polarity proteins cluster together to form three evolutionarily conserved polarity protein complexes, namely, Partitioning defective (Par) complex [Par3, Par6 and atypical protein kinase C (aPKC)], Crumbs complex [Crumbs, Pals and Pals1-associated tight junction protein (Patj)], and Scribble complex [Scribble (Scrib), discs large (Dlg) and lethal (2) giant larvae (Lgl)]. These polarity proteins influence cell polarity by interplaying with each 
other and other factors.

Proper cell polarity contributes to maintenance of tissue homeostasis. Loss of epithelial cell polarity has been widely assumed to be a key step in tissue disorganization and often lead to cancers [9]. In this review, we focus solely on the roles of Lgl in cell polarity and cancer development and discuss the potential mechanisms underlying the biological functions of Lgl.

\section{The conservative property of Lgl proteins}

Lgl is so named because it can influence larvae growth by inhibiting larval tissues from proliferation and differentiation. Loss of $\mathrm{Lgl}$ does not result in abnormal phenotypes of the Drosophila during its embryogenesis; however, the brain and the imaginal discs of the mutant larvae overgrow spectacularly and the resultant giant larvae die without entering metamorphosis [10]. Lgl proteins in different species have similar structures, which contain multiple WD40 domains and conserved phosphorylation sites in eukaryotes (Fig.1) [11, 12]. In human, members of Lgl family mainly include Lgl-1 and Lgl-2, also known as Hugl-1 and Hugl-2. The two have the similar structures, as shown in Fig 1. WD40 domains normally act as a scaffold for coordination of multiprotein complex assemblies; they are also involved in a wide variety of
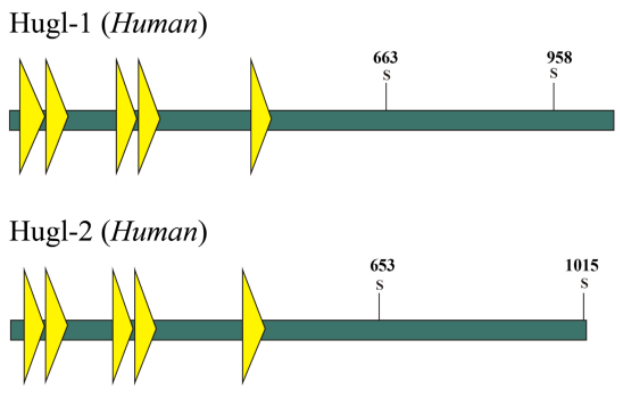

Mgl-1 (Mouse)
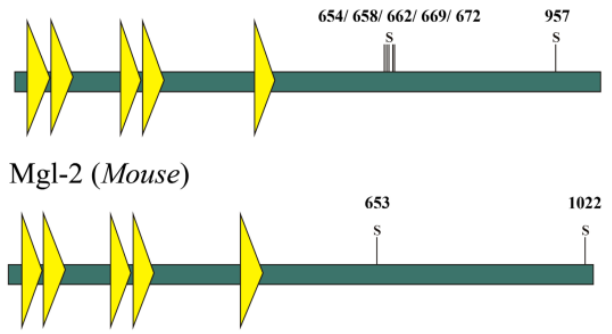

Rgl-1 (Rat)

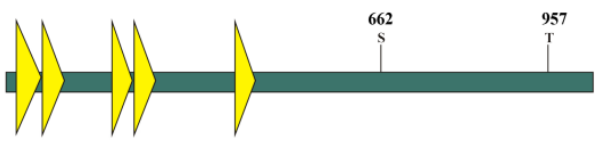

cell biological processes, including signal transduction, vesicle trafficking, cytoskeleton assembly and cell division [13, 14]. WD40 domains in Lgl have properties similar to those of cellular membranes and are probably involved in cell-cell interaction $[15,16]$. The WD40 domains spanning the N-terminal part of Lgl in Drosophila fold into two $\beta$-propellers, and then interact with the C-terminal part of Lgl [11]. At the C-terminus of Lgl exist conserved sites for serine phosphorylation, and the phosphorylation at these residues leads to an intramolecular interaction of the $\mathrm{N}$ - and C-terminal parts and alters the activity of $\mathrm{Lgl}$ $[11,17]$.

Proteins of the Lgl group have highly conserved structures and functions (Fig.2). Lgl in one species can be functionally substituted by homologues of $\mathrm{Lgl}$ from another species [18, 19]. Kim et al. used $M g l-1$, an lgl homologue in mouse, to complement the aberrant expressions of sro7 and sro77 ( $\mathrm{lgl}$ homologues in yeasts), and found that the loss of sro7/77 was associated with salt tolerance and temperature sensitivity and that sro7/77 could be functionally complemented by exogenous $\mathrm{Mgl}-1$ [12]. Furthermore, earlier studies showed yeast complementation with mammalian homologues [18]. Their research suggests a high level of functional conservation of $\lg l$ between different species.

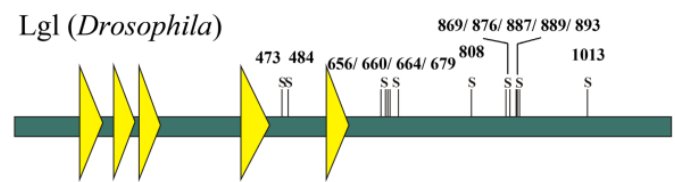

Lgl-1 (Xenopus)

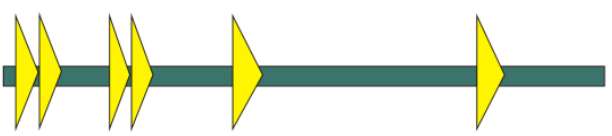

Lgl-2 (Xenopus)

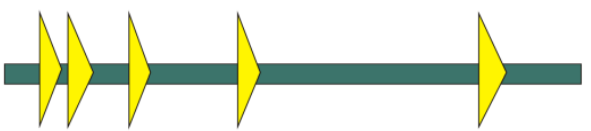

Sro7 (Yeast)

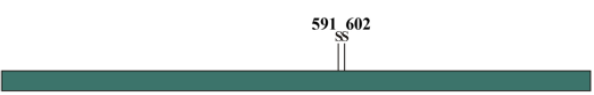

Sro77 (Yeast)

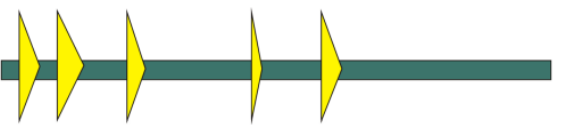

Figure 1. Schematic model of the domain structure of Lgl family proteins (Yeast, Xenopus, Drosophila, Mouse, Rat and Human). The domain structure was determined using the Uniprot (http://www.uniprot.org/) and SMART (http://smart.embl-heidelberg.de/) servers. A yellow triangle represents a WD40 domain. $S$ is a serine phosphorylation site. $T$ is a threonine phosphorylation site. 


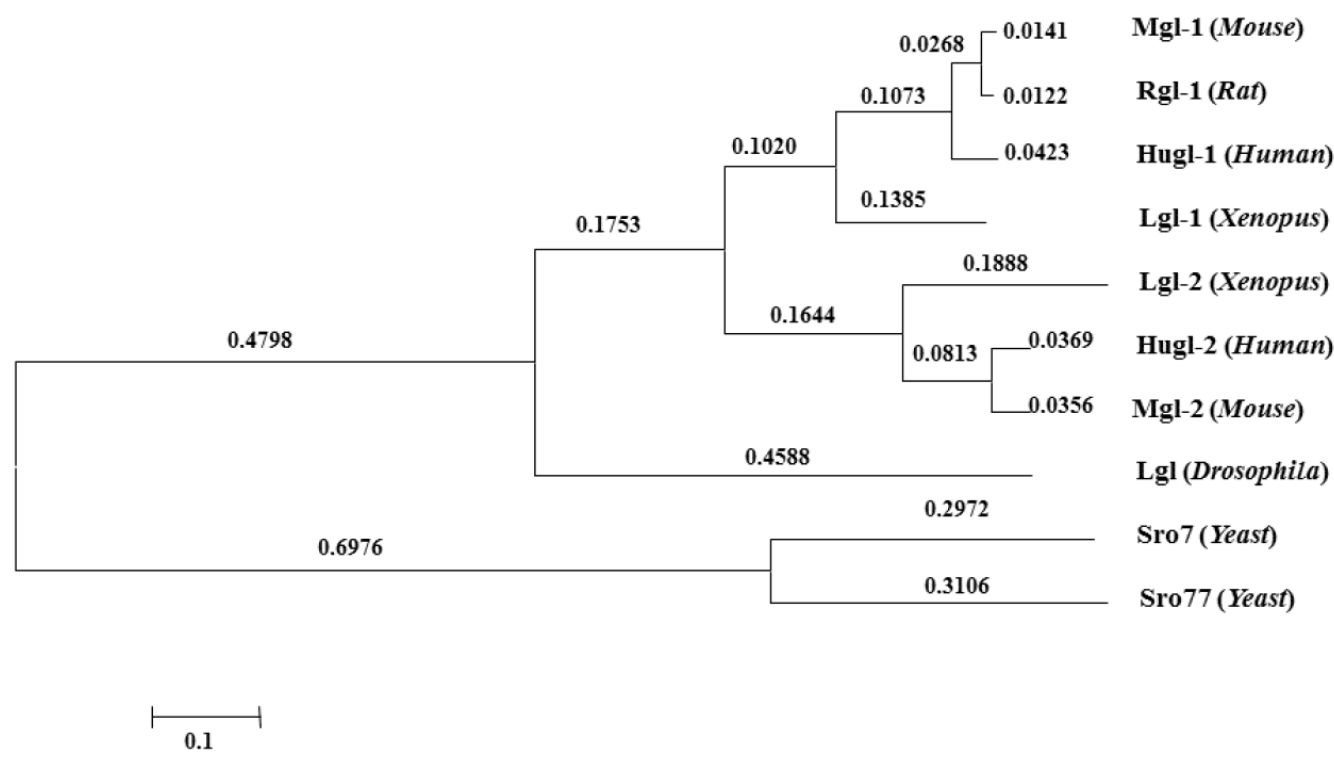

Figure 2. Conservation of $\mathrm{Lgl}$ family proteins in eukaryotes. Phylogenetic tree from the Macvector $7.2 \mathrm{ClustalW}$ multiple sequence alignment of $\mathrm{Lgl}$ proteins from Yeast (Sro7 and Sro77), Xenopus (Lgl-1 and Lgl-2), Drosophila (Lgl), Mouse (Mgl-1, Mgl-2), Rat (Rgl-1) and Human (Hugl-1 and Hugl-2). A value of 0.1 , as an approximate guide, corresponds to a $10 \%$ difference between two sequences.

\section{How Lgl works in organism models}

Lgl mutants are commonly utilized as genetic tools for investigation of the molecular basis of epithelial transformation and Lgl-induced tumor suppression. In vivo lgl-mutant organism models have been developed to determine the consequences of the aberrant expression of Lgl [20]. Lgl was originally identified in the Drosophila, which has long been recognized as an important genetically tractable model organism for elucidating the mechanism of tumorigenesis and tumor suppression [21-23]. In Drosophila, depletion of Lgl disturbs apico-basal cell polarity and alters cell proliferation, leading to malignant growth of larval brain neuroblasts and imaginal discs [24]. Lgl is distributed in the cytosol, and phosphorylated by aPKC in the apical domain, and recruited to the basolateral domain after cell-cell contact-initiated polarization [6, 25]; Lgl, interdependently binding to Dlg and Scribble as the Scribble complex [26], localizes to the basolateral domain of epithelial cells and excludes apical components from this region [24, 27, 28].

\section{The essential role of $\mathrm{Lgl}$ in cell polarity}

Although the molecular mechanism of how Lgl regulates cell polarity is not fully understood, studies using numerous model organisms have revealed the potential functions of Lgl. Defect of Lgl function, leading to a disruption of cell polarity and epithelial integrity, is associated with an increase in ectopic proliferation and tissue overgrowth $[29,30]$.

It is well-known that the Par, Crumbs, and Scribble complexes are necessary for establishment and maintenance of apico-basal cell polarity. As shown in figure 3, the Par and Crumbs complexes act in a mutually exclusive manner with the Scribble complex, and then the three complexes dynamically regulate polarization [27]. Lgl, as a member of the Scribble complex, can interplay with other polarity proteins. The Scribble complex has antagonistic interactions with the other two complexes, which is highlighted by the increased Crumbs or Par complex activity observed in Drosophila and mammalian cells with loss-of-function mutations in the three components of the Scribble complex [31]. The Crumbs complex and the Par complex can function mutually with Scribble complexes to define apical and basolateral surfaces (Fig.3), and the epithelial polarity cannot be maintained by the Crumbs complex individually in the absence of Lgl activity [31-33]. These findings indicate that $\mathrm{Lgl}$ plays an important role in maintaining the function integrity of the Scribble complex, and has certain influence on the interaction between the Scribble cell polarity complex and the others.

Lgl can also interplay with other polarity proteins individually (Fig.3). Lgl can be phosphorylated by aPKC [34]. After phosphorylation, Lgl becomes inactive and is released from the apical cell cortex and then recruited to the basolateral membrane by binding to Dlg to form a complex $[35,36]$. However, it is still not clear how this relocalization from the apical to the basolateral domain has occurred. As a competitor of Par3, Lgl can directly interact with Par6-aPKC protein complex in Drosophila polarizing neuroblasts and mammalian migrating cells $[6,37]$. By combining with the Par complex, Lgl can inhibit aPKC activity [38]. In addition to the interplay between the Scribble 
complex and the other two complexes via their components, the protein components of the Scribble complex also can interact with each other. A recent study has reported that phosphorylation of any one of the three conserved Ser residues situated in the central linker region of $\mathrm{Lgl}$ is enough to make $\mathrm{Lgl}$ bind to the Dlg guanylate kinase (GK) domain. This finding means that Dlg is able to directly interact with Lgl in a phosphorylation-dependent manner [36].

Interplay of Lgl with other polarity proteins is important to maintenance of cell biological and morphologic characteristics. Based on the above review, it can be considered that such interplay must be an essential way that $\mathrm{Lgl}$ affects cell polarity and other cell functions. Besides, $\lg l$ null mutation in Drosophila leads to an ectopic expression of Cyclin E (CycE), which is a key cell cycle regulator [29]. Therefore, Lg1 may modulate cell functions by relating other components, which has also been evidenced in mammalian Lgl proteins before. It is showed that Lgl-2 in mammalian cell lines can directly bind to p32, a kind of proteins which is related to the enhanced phosphorylation of aPKC on Lgl-2 and the regulation of cell polarity [39].

The critical function of Lgl in cell polarity may be represented by its ability to regulate polarized exocytosis, which is mostly evidenced by yeasts models.
Exocytosis regulates the transport of secreted and transmembrane macromolecules, including proteins and lipids, to the cell surface $[40,41]$. This transport is highly polarized and exquisitely regulated in order to maintain the molecular identities of the apical, lateral and basal membrane domains [42]. Lgl proteins regulate the polarized transport of exocytic vesicles, which is evidenced by the finding that cells with Sro7/77 (lgl homologues in yeast) mutants display prominent defects in exocytosis [43]. Sro7/77 is a downstream effector of Sec4, a Rab GTPase that mediates the post-Golgi stage of exocytosis [44]. Sro7/77 also affects exocytosis by directly interacting with a component of the exocyst complex, Exo84, which is essential for targeting vesicles to specific sites of the plasma membrane for exocytosis [42]. Moreover, in Madin-Darby canine kidney cells, the mammalian homologue of Lgl influences cell polarity via altering exocytosis, which may be due to an interaction with syntaxin 4, a t-SNARE protein (target soluble $\mathrm{N}$-ethylmaleimide attachment protein receptor) [25]. Thus, Lgl plays a role in exocytosis probably by binding to proteins such as the plasma membrane t-SNARE proteins and the components of the exocyst complex, or by regulating the pathways which mediate the fusion of post-Golgi transport vesicles to target membranes $[25,43]$.

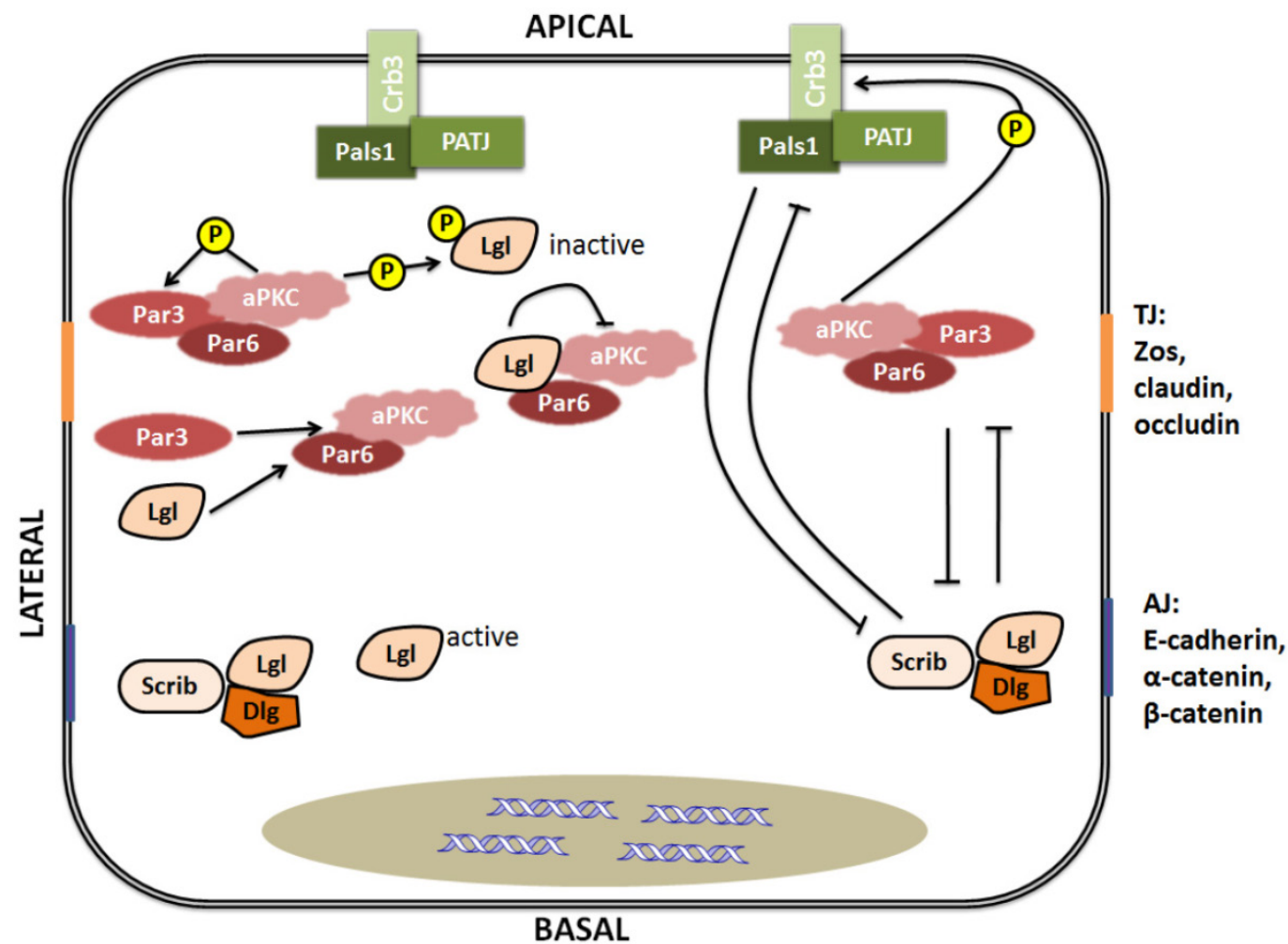

Figure 3. Interplay between polarity proteins. Left: Lgl interacts with other polarity proteins. Lgl can be phosphorylated by aPKC, and competes for Par3 in binding to Par6-aPKC. Right: Apico-basal polarity complexes dynamically regulate polarization by mutual exclusion. TJ is the abbreviation of tight junction [composed predominantly of ZOs (zona occludens), claudins and occludin]. AJ is the abbreviation of adherence junction (composed predominantly of E-cadherin, $\alpha$-catenin and $\beta$-catenin). 


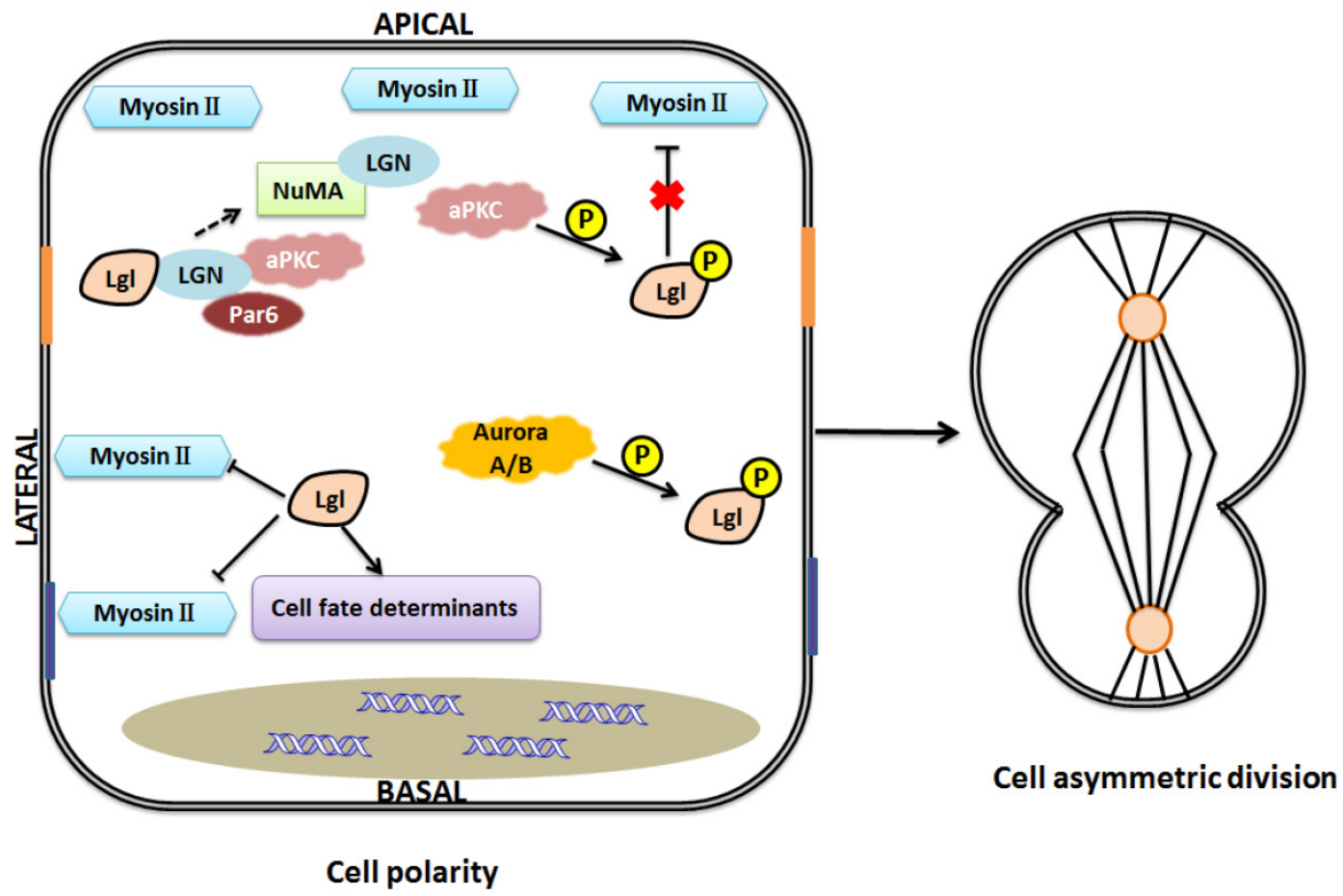

Figure 4. One model of Lgl function in the maintenance of cell polarity and asymmetric division. Lgl regulates cell fate determinants and interacts with LGN to influence the formation of LGN-NuMA complex. Nonphosphorylated Lgl negatively regulates myosin II activities at basolateral domain, and when Lgl is phosphorylated by aPKC at the apical domain, this inhibitive effect will be gone. Aurora A/B kinases can directly phosphorylate Lgl to influence its relocalization during mitosis. Asymmetric assembly of the actomyosin cytoskeleton and asymmetric distribution of cell fate determinants help maintain cell polarity and cell asymmetric division.

\section{Lgl regulates asymmetric cell divisions}

Asymmetric cell divisions are important regulators of stem cell and cancer biology. Various proteins, asymmetrically distributed during mitosis, are involved in the pathways underlying spindle orientation and asymmetric cell divisions, including polarity proteins and other cell fate determinants [45]. For example, in Drosophila neuroblasts, the activities of Par-3, Inscuteable (Insc), and Partner of inscuteable (Pins) are required for the spindle rotation [46]. Pins asymmetrically colocalizes with inscuteable in neuroblasts [46, 47]. G-protein signaling modulator 2 (GPSM2), also known as LGN (leu-gly-asn), the mammalian Pins homolog, plays a key role in spindle pole organization during mitosis in mammalian cells. NuMA, a nuclear mitotic apparatus protein, binds to LGN to form a complex $[48,49]$. Lgl-2 in mammal can directly bind to LGN to form a complex with aPKC, Par-6. This binding regulates the formation of LGN-NuMA complex, and then involves in the mitotic spindle organization and cell division [50].

Asymmetric cell divisions are regulated by the Par complex through phosphorylation of Lgl (Fig.4) [6]. Besides, recent studies showed that $\mathrm{Lgl}$ relocalizes to the cytoplasm during mitosis, and the mitotic $\mathrm{Au}-$ rora A/B kinases can directly phosphorylate Lgl to influence its relocalization in Drosophila [51, 52]. This provides a new insight into the regulation and function of Lgl in cell divisions. Moreover, Lgl is a component of the cytoskeletal network including non-muscle myosin II (NMII) heavy chain. As we know, cytoskeleton plays an essential role in cell division. Lgl affects cytoskeleton by directly interacting with NMII-A, an actin-based motor protein essential for various cell functions such as adhesion, migration, and polarity $[6,53,54]$. Lgl and Dlg regulate targeting of basal proteins such as Miranda, Prospero, Numb and Partner of Numb (Pon), which requires microfilament and myosin function [55]. Generally, unphosphorylated Lgl at the basolateral domain restricts activity of NMII-A and inhibits assembly of actin-myosin II cytoskeleton, while phosphorylated Lgl at the apical domain dissociates from the cytoskeleton, unable to influence the actin-myosin II cytoskeleton $[6,11]$. Lgl's regulation of cytoskeleton is involved in maintenance of the locations of cell fate determinants. For example, asymmetric segregation of Numb and Prospero, excluded from the apical cortex, is regulated by NMII-A cytoskeleton in Drosophila neuroblasts [56]. As we mentioned above, $\mathrm{Lgl}$ is a component of NMII cytoskeleton. The asymmetric activity of cytoskeleton caused by Lgl may contribute to maintenance of cell shape, cell polarity and normal cell division. Moreover, aPKC may inhibit the effects of Lgl on NMII and cytoskeleton since NMII and 
aPKC are considered to be competitive in binding to Lgl $[11,57]$. However, the inhibitive effect of Lgl on NMII has been challenged by the report that overexpression of Lgl leads to enhanced NMII activation [58]. The discrepancy might be explained as follows: Lgl overexpression might trigger the ability of cells to maintain their homeostasis, increase phosphorylation of Lgl by aPKC, and consequently, enhance NMII activation. However, this postulation remains to be confirmed in the future.

\section{How Lgl influences signaling pathways}

\section{The Hippo signaling pathway}

The Hippo signaling pathway is an evolutionarily conserved signaling network implicated in the proper control of cell survival and organ size. It coordinately promotes apoptosis and restricts cell proliferation, thus its deregulation can induce tumorous tissue overgrowth [59]. In Drosophila, the SWH (Sav-Wts-Hippo) signaling pathway mainly consists of the protein kinases Hippo (Hpo) and Warts (Wts), their respective adaptors Salvador (Sav) and Mats, the transcriptional co-factor Yorkie (Yki), as well as a number of upstream regulators, such as the cytoskeletal proteins Expanded (Ex) and Merlin (Mer) and the atypical cadherins Fat (Ft) and Dachsous (Ds) [60]. Figure $5 \mathrm{~A}$ shows the activation process of the SWH pathway in normal cells.

Because of the similar roles of $\mathrm{Lgl}$ and $\mathrm{SWH}$ pathway in regulating tissue growth and organ size, there must be a crucial relationship between Lgl and
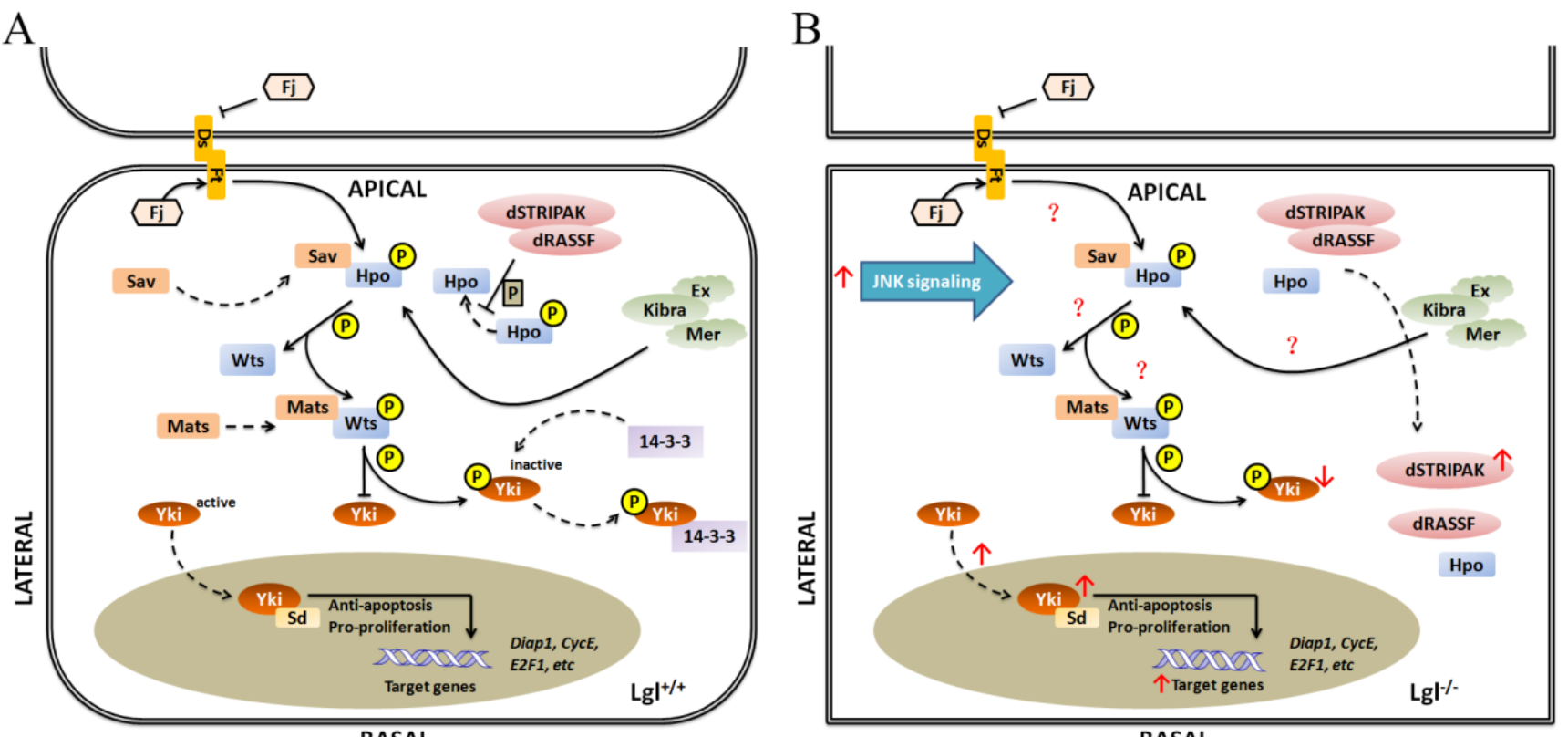

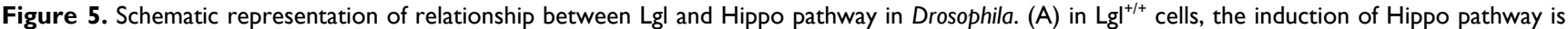
triggered; (B) in $\mathrm{Lg}^{-1 /-}$ cells, the down-regulation of Hippo pathway is occurred. Hpo tends to be more basolateral, and dRASSF and dSTRIPAK are mislocalized and colocalized with Hpo. The level of the phosphorylated Yki protein is downregulated, and Yki is translocated from the cytoplasm to the nucleus.
BASAL

the SWH pathway, which is evidenced by numerous studies. $\lg l$ null mutation suppresses cell death by upregulating the transcription and translation levels of downstream effectors of the SWH pathway, such as CycE, Diap1 (Drosophila inhibitor of apoptosis-1) [30, 38, 61]. Similarly, deregulation of the SWH pathway can upregulate the expression levels of its downstream effectors and inhibit cell death. The localizations and/or activities of SWH pathway components may be altered due to the different expression level of Lgl. Hpo mainly locates apicolaterally in normal cells but tends to be more basolateral as a result of loss of $\mathrm{lgl}$. In Drosophila, the Drosophila Striatin-interacting phosphatase and kinase (dSTRIPAK) directly combines with Drosophila Ras-association domain family protein dRASSF, dephosphorylates Hpo and prevents the activation of the SWH pathway to promote tissue growth. It has been found that dRASSF and dSTRIPAK are also mislocalized and colocalized with Hpo in $l g l$ null mutant tissues [38, 62]. Morover, $\operatorname{lgl}$ null mutation downregulates the level of the phosphorylated Yki protein and translocates Yki from the cytoplasm to the nucleus [29, 30]. The above-mentioned findings reveal that the absence of lgl is involved in the down-regulation of the $\mathrm{SWH}$ pathway. However, $\lg l$ null mutation does not change the localizations and expression levels of Ex and Ft at the apical cortex [38], suggesting that not all the components of the SWH pathway can be affected by lgl. 西 
Lgl has an antagonistic relationship with aPKC in regulation of the SWH pathway [38,62,63]. The overexpression of aPKC leads to colocalization of Hpo and RASSF in the basolateral domain of cells [38]. Besides, ectopic overactivity of aPKC may increase Yki activity through upregulation of Jun N-terminal kinase (JNK) signaling [64], just as the $l g l$ gene knockout does (Fig.5B).

\section{The Wnt signaling pathway}

The Wnt signaling consists of a large number of components, including the Wnt protein family, Wnt receptors the Frizzled (Fzd) family, and Dishevelled proteins (Dvl, essential mediators of Wnt signaling) [65]. The Wnt signaling is an important pathway for regulating cell polarity. Multiple lines of evidence have indicated that the Wnt signaling pathway is linked to numerous cell polarity proteins, including Lgl. For example, Dvl is required for maintenance of Lgl activity and localization at the cortex in Xenopus and Drosophila [66]. It has also been found that Fzd 8 receptor rather than Fzd 7 is implicated in modulation of $\mathrm{Lgl}$ activity and its delocalization from the basolateral cortex into the cytoplasm, which suggests that different subtypes of Fzd receptors may display distinct effects on Lgl [66]. As similar with Fzd receptors, Wnt proteins may also have distinct effects due to their different ligands. Moreover, previous work has shown that Wnt5a causes the instability of Lgl, dissociating Lgl from the cell cortex in Xenopus [67]. The molecular connection between Wnt5a and Lgl is essential for morphological and molecular changes of cell polarity construction. The above review indicates that the Wnt signaling may impact Lgl by changing its location, activity and consequent function.

\section{The Notch signaling pathway}

The Notch signaling is an evolutionarily conserved pathway. Previous research has shown that Lgl affects the Notch signaling through controlling the localizations of the Notch signaling regulators, such as the asymmetric segregation of Numb, asymmetric localization of Neuralized, and plasma-membrane localization of Sanpodo [8, 68]. lgl depletion upregulates the Notch signaling in Drosophila eye tissue [69]. In addition, $\lg l$ mutant mice exhibit hyperplasia of the neural tubes, linked with the enhanced Notch signaling [70]. The numb null mice also undergo hyperplasia in the developing cortex [70, 71]. Asymmetric cell divisions promote Notch-dependent epidermal differentiation [72]. It is assumed that abnormal expression of $l g l$ induces ectopic asymmetric cell division and deregulation of the Notch signaling pathway through regulating components of the Notch signaling.

Besides the three signaling pathways we have described above, Lgl may have relationships with other signaling pathways, such as the mitochondria related signaling, Ras signaling, and mTOR signaling. However, little is known about the role of $\mathrm{Lgl}$ in these signaling patheways, which may present interesting topics for future research.

\section{The role of Lgl in human}

Normal polarity signaling is required for maintenance of tissue integrity and disordered cell polarity may contribute to development of epithelial-to-mesenchymal transition (EMT) and tumorigenesis [73-75]. Since polarity proteins have important roles in regulating cell polarity, they are generally considered as modulators of tumorigenesis [76, 77]. In mice, lgl-1 gene knockout results in the hyperplasia of the neural tubes, forming structures similar to the neuroblastic rosettes in human primitive neuroectodermal tumors. Severe hydrocephalus and neonatal death are also observed in $l g l 1 \%$ mouse pups [70]. Loss of Lgl leads to severe malignant phenotypes in organism models. Therefore, what roles Lgl plays in human is need to be discovered.

The human homologues of $\mathrm{Lgl}$ are known as Hugl-1 and Hugl-2 [78]. Hugl proteins contain several conserved functional domains highly homologous to some regions of $\mathrm{Lgl}$, which indicates that Hugl and Lgl proteins may have closely related functions [19, 53]. Accumulating evidence has shown that Hugl plays a suppressive role in various human epithelial cancers. In hepatocelluar carcinoma (HCC), Hugl-1 transcripts are frequently mutated by aberrant splicing, indicating that Hugl-1 mutation may be involved in progression of HCC [79]. In esophageal carcinoma, Hugl-1 inhibits cell proliferation and promotes apoptosis via regulation of the mitochondria-related pathway [80]. In malignant melanoma, Hugl-1 expression varies with the progression or development of the disease and is involved in increased cell adhesion and decreased cell migration, suggesting that loss of Hugl-1 expression contributes to melanoma progression [81]. In colorectal cancer, reduced expression of Hugl-1 is implicated in the progression of the disease [82]. However, studies of Lgl in leukemia have displayed inconsistent findings. Heidel et al. have reported that loss of Hugl-1 enhances self-renewal and fitness of hematopoietic stem cells (HSCs) and is associated with poor prognosis in patients with acute myeloid leukemia (AML) [83]. On the other hand, a recent study has shown that the absence of $\operatorname{lgll}$ in mice does not alter leukemia driven by constitutive Notch, c-Myc or Jak2 signaling [84]. The discrepancy may be due to the different cellular contexts of haematopoietic system, and this indicates that the role of Lgl-1 in regulation of leukemia might be restricted to 
specific cell conditions. The tumor suppressive role of Hugl-1 may be limited to specific cellular contexts of different cancer types.

Hugl-2 is also associated with cancer progression and suppression. It has been found that Snail, an EMT inducer, downregulate Hugl-2 expression in cells. However, when Hugl-2 expression is induced in Snail-expressing cells, Hugl-2 overrides Snail tumorigenesis and induces mesenchymal to epithelial transition (MET) [85]. Another research has also found that ZEB1, an EMT-inducing transcriptional repressor, suppresses the expression of Hugl-2 in colorectal and breast cancers [86]. ZEB1 and Snail have the ability of regulating the expression of the cell-cell adhesion molecule and thereby controlling EMT progression. Increased ZEB1 and Snail expression levels, involving with dedifferentiation and invasion of tumor cells, can be observed in different human cancers, correlating with dedifferentiation and invasion of tumor cells [87-89]. These findings indicate that loss of Hugl-2 is associated with EMT, and EMT-related factors have negative effects on the expression of Hugl-2. Moreover, Hugl-1 and Hugl-2 are both essential for maintenance of polarity, proliferation and morphology of human mammary epithelial cells [90]. Aberrant localization or deletion of Lgl2 contributes to gastric epithelial dysplasia and gastric adenocarcinoma as well as pancreatic intraepithelial neoplasia and pancreatic ductal adenocarcinoma [91-93]. Apical membrane localization of Hugl-2, combined with aPKC, is associated with lymphatic invasion and lymph node metastasis in human lung adenocarcinoma [94]. However, roles of Hugl-2 in regulation of other cancer types need to be studied in future works. Taken together, the expression changes of Hugl-1/-2 in various cancers suggest their tumor-suppressive roles (Table 1).

Previous studies, reviewed above, have suggested the suppressive role of Hugl in cancer progression, however, the exact mechanism underlying this suppression effect still has not elucidated. Com- bined with the influences of Lgl on cell polarity mentioned above, we postulate that similarly to Drosophila or yeasts or other species, the effects of Hugl on human cancer progression must be based on its role of regulating cell normal functioning, and be associated with its mislocalization, deficit of expression, and dysregulation of its downstream pathways. Further investigations are needed to be clarified the exact mechanism underlying cancer suppressive effect of Hugl.

\section{Conclusion and perspectives}

We have attempted to summarize the cell biological functions of Lgl. As outlined above, $\mathrm{Lgl}$ affects epithelial cell polarity and cell proliferation in various manners, including combining with other proteins, regulating exocytosis, modulating cytoskeleton, and being implicated in signaling pathways. Drosophila, yeasts, mice, etc. are used as model organism for studies of Lgl and some mechanisms for Lgl functions in these species have been reported in literatures. However, little is known about the mechanism for Lgl regulation of epithelial cell polarity and cell division in human. It is still uncertain what Lgl proteins do exactly, how they are translocated, how their interactions with other proteins are regulated, how their participation in various signaling pathways are mediated, and whether the alteration of Lgl in human are similar to those in other species.

Lgl dysfunction or depletion inevitably has a broad impact on cancers. Lgl may act as a neoplastic tumor suppressor in numerous species. However, the exact tumor-suppressing mechanism of Lgl is still unknown. In short, unraveling the mechanisms responsible for the effects of Lgl alteration on tumorigenesis is the major goal of the future work. A better and deeper understanding of the mechanisms underlying the changes in Lgl and the pathways it participates in is likely to provide clues for developing new ways to diagnose and control cancers.

Table 1. Alterations in Hugl expression and location in human cancers

\begin{tabular}{|c|c|c|c|}
\hline Gene / Protein & Alteration & Tumor type & Phenotype \\
\hline \multirow[t]{5}{*}{ Hugl-1 } & Downregulation & $\begin{array}{l}\text { Breast, melanoma, prostate, lung, and ovarian } \\
\text { tumours }[81,90]\end{array}$ & $\begin{array}{l}\text { Malignant phenotype, disruption of cell polarity and tissue archi- } \\
\text { tecture, overgrowth }\end{array}$ \\
\hline & Downregulation & Colon and colorectal cancer [82] & Advanced stage, lymph node metastases \\
\hline & Aberrantly splicing & Hepatocellular carcinoma [79] & Poor differentiation, large tumour size \\
\hline & Null mutant & Acute myeloid leukemia [83] & Poor prognosis, enhanced hematopoietic stem cells self-renewal \\
\hline & Upregulation & Esophageal carcinoma [80] & Induced cell apoptosis \\
\hline \multirow[t]{4}{*}{ Hugl-2 } & Upregulation & Human cell lines $[85,86]$ & MET \\
\hline & $\begin{array}{l}\text { Mislocalization or } \\
\text { downregulation }\end{array}$ & $\begin{array}{l}\text { Gastric epithelial dysplasia, gastric adenocarcino- } \\
\text { ma, pancreatic intraepithelial neoplasia and pan- } \\
\text { creatic ductal adenocarcinoma [91-93] }\end{array}$ & Disruption of tissue morphology \\
\hline & Mislocalization & Lung adenocarcinoma [94] & Lymphatic invasion and lymph node metastases \\
\hline & Null mutant & Breast cancer [90] & Increased proliferation and disruption of cell polarity \\
\hline
\end{tabular}




\section{Competing Interests}

The authors have declared that no competing interest exists.

\section{References}

1. Etienne-Manneville S. Polarity proteins in migration and invasion. Oncogene. 2008; 27: 6970-80.

2. Martin-Belmonte F, Perez-Moreno M. Epithelial cell polarity, stem cells and cancer. Nature Reviews Cancer. 2012; 12: 23-38.

3. Rodriguez-Boulan E, Macara IG. Organization and execution of the epithelial polarity programme. Nature reviews Molecular cell biology. 2014; 15: 225-42.

4. Gibson MC, Perrimon N. Apicobasal polarization: epithelial form and function. Curr Opin Cell Biol. 2003; 15: 747-52.

5. Sabherwal N, Papalopulu N. Apicobasal polarity and cell proliferation during development. Essays Biochem. 2012; 53: 95-109.

6. Betschinger J, Mechtler K, Knoblich JA. The Par complex directs asymmetric cell division by phosphorylating the cytoskeletal protein Lgl. Nature. 2003; 422: 326-30

7. Wodarz A. Molecular control of cell polarity and asymmetric cell division in Drosophila neuroblasts. Curr Opin Cell Biol. 2005; 17: 475-81.

8. Langevin J, Le Borgne R, Rosenfeld F, Gho M, Schweisguth F, Bellaiche Y. Lethal giant larvae controls the localization of notch-signaling regulators numb, neuralized, and Sanpodo in Drosophila sensory-organ precursor cells. Curr Biol. 2005; 15: 955-62.

9. Wodarz A, Gonzalez C. Connecting cancer to the asymmetric division of stem cells. Cell. 2006; 124: 1121-3.

10. De Lorenzo C, Strand D, Mechler BM. Requirement of Drosophila I(2)gl function for survival of the germline cells and organization of the follicle cells in a columnar epithelium during oogenesis. Int J Dev Biol. 1999; 43: 207-17.

11. Betschinger J, Eisenhaber F, Knoblich JA. Phosphorylation-induced autoinhibition regulates the cytoskeletal protein Lethal (2) giant larvae. Current Biology. 2005; 15: 276-82.

12. Kim Y-K, Kim Y-S, Baek K-H. The WD-40 repeat motif of Lgl tumor suppressor proteins associated with salt tolerance and temperature sensitivity. Biochemical and biophysical research communications. 2005; 331: 922-8.

13. Smith TF, Gaitatzes C, Saxena K, Neer EJ. The WD repeat: a common architecture for diverse functions. Trends in biochemical sciences. 1999; 24: 181-5.

14. Stirnimann CU, Petsalaki E, Russell RB, Muller CW. WD40 proteins propel cellular networks. Trends Biochem Sci. 2010; 35: 565-74.

15. Lützelschwab R, Klämbt C, Rossa R, Schmidt O. A protein product of the Drosophila recessive tumor gene, 1 (2) giant gl, potentially has cell adhesion properties. The EMBO journal. 1987; 6: 1791.

16. Yamanaka T, Ohno S. Role of $\mathrm{Lgl} / \mathrm{Dlg} / \mathrm{Scribble}$ in the regulation of epithelial junction, polarity and growth. Frontiers in bioscience: a journal and virtual library. 2008; 13: 6693.

17. Graybill C, Prehoda KE. Ordered multisite phosphorylation of lethal giant larvae by atypical protein kinase C. Biochemistry. 2014; 53: 4931-7.

18. Larsson K, Böhl F, Sjöström I, Akhtar N, Strand D, Mechler BM, et al. The Saccharomyces cerevisiae SOP1 andSOP2 Genes, Which Act in Cation Homeostasis, Can Be Functionally Substituted by the Drosophila lethal (2) giant larvae Tumor Suppressor Gene. J Biol Chem. 1998; 273: 33610-8.

19. Grifoni D, Garoia F, Schimanski CC, Schmitz G, Laurenti E, Galle PR, et al. The human protein Hugl-1 substitutes for Drosophila lethal giant larvae tumour suppressor function in vivo. Oncogene. 2004; 23: 8688-94.

20. Froldi F, Ziosi M, Tomba G, Parisi F, Garoia F, Pession A, et al. Drosophila lethal giant larvae neoplastic mutant as a genetic tool for cancer modeling. Current genomics. 2008; 9: 147-54.

21. Watson KL, Justice RW, Bryant PJ. Drosophila in cancer research: the first fifty tumor suppressor genes. J Cell Sci. 1994; 1994: 19-33.

22. Brumby AM, Richardson HE. Using Drosophila melanogaster to map human cancer pathways. Nature Reviews Cancer. 2005; 5: 626-39.

23. Hadorn E. Die Degeneration der Imaginalscheiben bei letalen Drosophila-Larven der Mutation Lethal-giant. Rev suisse zool. 1938; 45: 425-9.

24. Bilder D, Li M, Perrimon N. Cooperative regulation of cell polarity and growth by Drosophila tumor suppressors. Science. 2000; 289: 113-6.

25. Mu A, Cohen D, Yeaman C, Nelson WJ, Rodriguez-Boulan E, Brennwald PJ. Mammalian homolog of Drosophila tumor suppressor lethal (2) giant larvae interacts with basolateral exocytic machinery in Madin-Darby canine kidney cells. Mol Biol Cell. 2002; 13: 158-68.

26. Humbert P, Russell S, Richardson H. Dlg, Scribble and Lgl in cell polarity, cell proliferation and cancer. BioEssays : news and reviews in molecular, cellular and developmental biology. 2003; 25: 542-53

27. Ellenbroek SIJ, Iden S, Collard JG. Cell polarity proteins and cancer. Semin Cancer Biol. 2012; 22: 208-15.

28. St Johnston D, Ahringer J. Cell polarity in eggs and epithelia: parallels and diversity. Cell. 2010; 141: 757-74.

29. Grzeschik NA, Amin N, Secombe J, Brumby AM, Richardson HE. Abnormalities in cell proliferation and apico-basal cell polarity are separable in Drosophila lgl mutant clones in the developing eye. Dev Biol. 2007; 311: $106-23$
30. Grzeschik NA, Parsons LM, Richardson HE. Lgl, the SWH pathway and tumorigenesis. Cell Cycle. 2010; 9: 3202-12.

31. Humbert PO, Dow LE, Russell SM. The Scribble and Par complexes in polarity and migration: friends or foes? Trends Cell Biol. 2006; 16: 622-30.

32. Bilder D, Schober M, Perrimon N. Integrated activity of PDZ protein complexes regulates epithelial polarity. Nat Cell Biol. 2003; 5: 53-8.

33. Tanentzapf $G$, Tepass $U$. Interactions between the crumbs, lethal giant larvae and bazooka pathways in epithelial polarization. Nat Cell Biol. 2003; 5: 46-52.

34. Tian A-G, Deng W-M. Lgl and its phosphorylation by aPKC regulate oocyte polarity formation in Drosophila. Development. 2008; 135: 463-71.

35. Hutterer A, Betschinger J, Petronczki M, Knoblich JA. Sequential roles of Cdc42, Par-6, aPKC, and Lgl in the establishment of epithelial polarity during Drosophila embryogenesis. Dev Cell. 2004; 6: 845-54.

36. Zhu J, Shang Y, Wan Q, Xia Y, Chen J, Du Q, et al. Phosphorylation-dependent interaction between tumor suppressors Dlg and Lgl. Cell research. 2014.

37. Plant PJ, Fawcett JP, Lin DC, Holdorf AD, Binns K, Kulkarni S, et al. A polarity complex of mPar-6 and atypical PKC binds, phosphorylates and regulates mammalian Lgl. Nat Cell Biol. 2003; 5: 301-8.

38. Grzeschik NA, Parsons LM, Allott ML, Harvey KF, Richardson HE. Lgl, aPKC, and Crumbs regulate the Salvador/Warts/Hippo pathway through two distinct mechanisms. Current Biology. 2010; 20: 573-81.

39. Bialucha CU, Ferber EC, Pichaud F, Peak-Chew SY, Fujita Y. p32 is a novel mammalian $\mathrm{Lgl}$ binding protein that enhances the activity of protein kinase $\mathrm{C}$ zeta and regulates cell polarity. Journal of Cell Biology. 2007; 178: 575-81.

40. Hsu S-C, TerBush D, Abraham M, Guo W. The exocyst complex in polarized exocytosis. International review of cytology. 2004; 233: 243-65.

41. He B, Guo W. The exocyst complex in polarized exocytosis. Curr Opin Cell Biol. 2009; 21: 537-42.

42. Zhang X, Wang P, Gangar A, Zhang J, Brennwald P, TerBush D, et al. Lethal giant larvae proteins interact with the exocyst complex and are involved in polarized exocytosis. The Journal of cell biology. 2005; 170: 273-83.

43. Lehman K, Rossi G, Adamo JE, Brennwald P. Yeast homologues of tomosyn and lethal giant larvae function in exocytosis and are associated with the plasma membrane SNARE, Sec9. The Journal of cell biology. 1999; 146: 125-40.

44. Grosshans BL, Andreeva A, Gangar A, Niessen S, Yates JR, Brennwald P, et al. The yeast lgl family member Sro7p is an effector of the secretory Rab GTPase Sec4p. The Journal of cell biology. 2006; 172: 55-66.

45. Neumuller RA, Knoblich JA. Dividing cellular asymmetry: asymmetric cell division and its implications for stem cells and cancer. Genes Dev. 2009; 23: 2675-99.

46. Yu F, Morin X, Cai Y, Yang X, Chia W. Analysis of partner of inscuteable, a novel player of Drosophila asymmetric divisions, reveals two distinct steps in inscuteable apical localization. Cell. 2000; 100: 399-409.

47. Kraut R, Chia W, Jan LY, Jan YN, Knoblich JA. Role of inscuteable in orienting asymmetric cell divisions in Drosophila. Nature. 1996; 383: 50-5.

48. Du Q, Stukenberg PT, Macara IG. A mammalian Partner of inscuteable binds NuMA and regulates mitotic spindle organization. Nat Cell Biol. 2001; 3: 1069-75.

49. Du Q, Taylor L, Compton DA, Macara IG. LGN blocks the ability of NuMA to bind and stabilize microtubules. A mechanism for mitotic spindle assembly regulation. Curr Biol. 2002; 12: 1928-33.

50. Yasumi M, Sakisaka T, Hoshino T, Kimura T, Sakamoto Y, Yamanaka T, et al Direct binding of Lgl2 to LGN during mitosis and its requirement for normal cell division. J Biol Chem. 2005; 280: 6761-5.

51. Bell GP, Fletcher GC, Brain R, Thompson BJ. Aurora kinases phosphorylate lgl to induce mitotic spindle orientation in Drosophila epithelia. Curr Biol. 2015; 25: 61-8.

52. Carvalho CA, Moreira S, Ventura G, Sunkel CE, Morais-de-Sa E. Aurora A Triggers Lgl Cortical Release during Symmetric Division to Control Planar Spindle Orientation. Curr Biol. 2015; 25: 53-60.

53. Strand D, Jakobs R, Merdes G, Neumann B, Kalmes A, Heid HW, et al. The Drosophila lethal (2) giant larvae tumor suppressor protein forms homo-oligomers and is associated with nonmuscle myosin II heavy chain. The Journal of cell biology. 1994; 127: 1361-73.

54. Kalmes A, Merdes G, Neumann B, Strand D, Mechler BM. A serine-kinase associated with the p127-l (2) gl tumour suppressor of Drosophila may regulate the binding of p127 to nonmuscle myosin II heavy chain and the attachment of p127 to the plasma membrane. J Cell Sci. 1996; 109: 1359-68.

55. Peng C-Y, Manning L, Albertson R, Doe CQ. The tumour-suppressor genes lgl and $\mathrm{dlg}$ regulate basal protein targeting in Drosophila neuroblasts. Nature. 2000; 408: 596-600.

56. Barros CS, Phelps CB, Brand AH. Drosophila Nonmuscle Myosin II Promotes the Asymmetric Segregation of Cell Fate Determinants by Cortical Exclusion Rather Than Active Transport. Dev Cell. 2003; 5: 829-40.

57. Dahan I, Petrov D, Cohen-Kfir E, Ravid S. The tumor suppressor Lgl1 forms discrete complexes with NMII-A and Par6a-aPKC 3 that are affected by Lgl1 phosphorylation. J Cell Sci. 2014; 127: 295-304.

58. Wan Q, Liu J, Zheng Z, Zhu H, Chu X, Dong Z, et al. Regulation of myosin activation during cell-cell contact formation by Par3-Lgl antagonism: entosis without matrix detachment. Mol Biol Cell. 2012; 23: 2076-91.

59. Pan D. The hippo signaling pathway in development and cancer. Dev Cell. 2010; 19: 491-505.

60. Alice G, Nicolas T. The Hippo pathway and apico-basal cell polarity. Biochemical Journal. 2011; 436: 213-24 
61. Humbert P, Grzeschik N, Brumby A, Galea R, Elsum I, Richardson H. Control of tumourigenesis by the Scribble/Dlg/Lgl polarity module. Oncogene. 2008; 27: 6888-907.

62. Parsons LM, Grzeschik NA, Richardson HE. lgl Regulates the Hippo Pathway Independently of Fat/Dachs, Kibra/Expanded/Merlin and dRASSF/dSTRIPAK. Cancers (Basel). 2014; 6: 879-96.

63. Grifoni D, Froldi F, Pession A. Connecting epithelial polarity, proliferation and cancer in Drosophila: the many faces of lgl loss of function. Int J Dev Biol. 2013; 57: 677-87

64. Sun G, Irvine KD. Regulation of Hippo signaling by Jun kinase signaling during compensatory cell proliferation and regeneration, and in neoplastic tumors. Dev Biol. 2011; 350: 139-51.

65. Clevers H, Nusse R. Wnt/beta-catenin signaling and disease. Cell. 2012; 149: 1192-205.

66. Dollar GL, Weber U, Mlodzik M, Sokol SY. Regulation of Lethal giant larvae by Dishevelled. Nature. 2005; 437: 1376-80.

67. Choi SC, Sokol SY. The involvement of lethal giant larvae and Wnt signaling in bottle cell formation in Xenopus embryos. Dev Biol. 2009; 336: 68-75.

68. Roegiers F, Jan LY, Jan YN. Regulation of membrane localization of sanpodo by lethal giant larvae and neuralized in asymmetrically dividing cells of Drosophila sensory organs. Mol Biol Cell. 2005; 16: 3480-7.

69. Parsons LM, Portela M, Grzeschik NA, Richardson HE. Lgl Regulates Notch Signaling via Endocytosis, Independently of the Apical aPKC-Par6-Baz Polarity Complex. Curr Biol. 2014; 24: 2073-84.

70. Klezovitch O, Fernandez TE, Tapscott SJ, Vasioukhin V. Loss of cell polarity causes severe brain dysplasia in Lgl1 knockout mice. Genes Dev. 2004; 18 : 559-71.

71. Li HS, Wang D, Shen Q, Schonemann MD, Gorski JA, Jones KR, et al. Inactivation of Numb and Numblike in embryonic dorsal forebrain impairs neurogenesis and disrupts cortical morphogenesis. Neuron. 2003; 40: 1105-18.

72. Williams SE, Beronja S, Pasolli HA, Fuchs E Asymmetric cell divisions promote Notch-dependent epidermal differentiation. Nature. 2011; 470: 353-8.

73. Shin K, Fogg VC, Margolis B. Tight junctions and cell polarity. Annu Rev Cell Dev Biol. 2006; 22: 207-35.

74. Nakaya Y, Sheng G. EMT in developmental morphogenesis. Cancer letters. 2013; 341: 9-15.

75. Casarsa C, Bassani N, Ambrogi F, Zabucchi G, Boracchi P, Biganzoli E, et al. Epithelial-to-mesenchymal transition, cell polarity and stemness-associated features in malignant pleural mesothelioma. Cancer Lett. 2011; 302: 136-43.

76. Gardiol D, Zacchi A, Petrera F, Stanta G, Banks L. Human discs large and scrib are localized at the same regions in colon mucosa and changes in their expression patterns are correlated with loss of tissue architecture during malignant progression. International Journal of Cancer. 2006; 119: 1285-90.

77. Subbaiah VK, Narayan N, Massimi P, Banks L. Regulation of the DLG tumor suppressor by beta-catenin. International journal of cancer Journal international du cancer. 2012; 131: 2223-33.

78. Strand D, Unger S, Corvi R, Hartenstein K, Schenkel H, Kalmes A, et al. A human homologue of the Drosophila tumour suppressor gene l (2) gl maps to 17p11. 2-12 and codes for a cytoskeletal protein that associates with nonmuscle myosin II heavy chain. Oncogene. 1995; 11: 291-301.
79. Lu X, Feng X, Man X, Yang G, Tang L, Du D, et al. Aberrant splicing of Hugl-1 is associated with hepatocellular carcinoma progression. Clin Cancer Res. 2009; 15: 3287-96.

80. Song J, Peng X-L, Ji M-Y, Ai M-H, Zhang J-X, Dong W-G. Hugl-1 induces apoptosis in esophageal carcinoma cells both in vitro and in vivo. World journal of gastroenterology: WJG. 2013; 19: 4127.

81. Kuphal S, Wallner S, Schimanski C, Bataille F, Hofer P, Strand S, et al. Expression of Hugl-1 is strongly reduced in malignant melanoma. Oncogene. 2006; 25: 103-10.

82. Schimanski CC, Schmitz G, Kashyap A, Bosserhoff AK, Bataille F, Schäfer SC, et al. Reduced expression of Hugl-1, the human homologue of Drosophila tumour suppressor gene $\operatorname{lgl}$, contributes to progression of colorectal cancer. Oncogene. 2005; 24: 3100-9.

83. Heidel FH, Bullinger L, Arreba-Tutusaus P, Wang Z, Gaebel J, Hirt C, et al. The cell fate determinant Llgl1 influences HSC fitness and prognosis in AML. J Exp Med. 2013; 210: 15-22.

84. Hawkins ED, Oliaro J, Ramsbottom KM, Ting SB, Sacirbegovic F, Harvey M, et al. Lethal Giant Larvae 1 Tumour Suppressor Activity Is Not Conserved in Models of Mammalian T and B Cell Leukaemia. PloS one. 2014; 9: e87376.

85. Kashyap A, Zimmerman T, Ergül N, Bosserhoff A, Hartman U, Alla V, et al. The human Lgl polarity gene, Hugl-2, induces MET and suppresses Snail tumorigenesis. Oncogene. 2013; 32: 1396-407.

86. Spaderna S, Schmalhofer O, Wahlbuhl M, Dimmler A, Bauer K, Sultan A, et al. The transcriptional repressor ZEB1 promotes metastasis and loss of cell polarity in cancer. Cancer research. 2008; 68: 537-44.

87. Browne G, Sayan AE, Tulchinsky E. ZEB proteins link cell motility with cell cycle control and cell survival in cancer. Cell Cycle. 2010; 9: 886-91.

88. Wu Y, Zhou BP. TNF-alpha/NF-kappaB/Snail pathway in cancer cell migration and invasion. British journal of cancer. 2010; 102: 639-44.

89. Wu YD, Zhou BHP. Snail More than EMT. Cell adhesion \& migration. 2010; 4: 199-203.

90. Russ A, Louderbough JM, Zarnescu D, Schroeder JA. Hugl1 and Hugl2 in mammary epithelial cells: polarity, proliferation, and differentiation. PloS one. 2012; 7: e47734.

91. Lisovsky M, Dresser K, Baker S, Fisher A, Woda B, Banner B, et al. Cell polarity protein Lgl2 is lost or aberrantly localized in gastric dysplasia and adenocarcinoma: an immunohistochemical study. Modern Pathology. 2009; 22: 977-84.

92. Lisovsky M, Dresser K, Woda B, Mino-Kenudson M. Immunohistochemistry for cell polarity protein lethal giant larvae 2 differentiates pancreatic intraepithelial neoplasia-3 and ductal adenocarcinoma of the pancreas from lower-grade pancreatic intraepithelial neoplasias. Human Pathology. 2010; 41: 902-9.

93. Lisovsky M, Ogawa F, Dresser K, Woda B, Lauwers GY. Loss of cell polarity protein Lgl2 in foveolar-type gastric dysplasia: correlation with expression of the apical marker aPKC-zeta. Virchows Archiv. 2010; 457: 635-42.

94. Imamura N, Horikoshi Y, Matsuzaki T, Toriumi K, Kitatani K, Ogura G, et al. Localization of aPKC Lambda/Iota and Its Interacting Protein, Lgl2, Is Significantly Associated with Lung Adenocarcinoma Progression. The Tokai journal of experimental and clinical medicine. 2012; 38: 146-58. 